\title{
Analyse épidémiologique de l'émergence et de la disparition du variant Kappa du SRAS-CoV-2 dans une région de la Colombie-Britannique, Canada
}

\author{
Cher Ghafari ${ }^{\star \star}$, Michael Benusic ${ }^{1}$, Natalie Prystajecky², Hind Sbihi², Kimia Kamelian², Linda Hoang ${ }^{2}$
}

\section{Résumé}

Contexte : Le variant Kappa est désigné comme un variant d'intérêt ( $\mathrm{VI}$ ) du coronavirus du syndrome respiratoire aigu sévère 2 (SRAS-CoV-2). Nous avons recensé 195 cas du variant Kappa dans une région de la Colombie-Britannique, au Canada, le plus grand agrégat spatio-temporel publié en Amérique du Nord.

Objectifs : Décrire l'épidémiologie du variant Kappa en relation avec d'autres variants préoccupants (VP) du SRAS-CoV-2 en circulation dans la région afin de déterminer si l'épidémiologie du variant Kappa demande un statut de VI ou de VP.

Méthodes : Les échantillons cliniques qui se sont avérés positifs pour le SRAS-CoV-2 collectés entre le 10 mars et le 2 mai 2021 ont été examinés pour la détection des VP circulants connus; environ $50 \%$ des échantillons ont ensuite été sélectionnés pour le séquençage du génome entier. Une analyse épidémiologique a été réalisée en comparant les caractéristiques des cas Kappa aux principaux variants circulant dans la région (Alpha et Gamma) et aux cas non VP/VI.

Résultats : Au total, 2079 cas de maladie à coronavirus 2019 (COVID-19) ont été signalés dans la région pendant la période d'étude, dont $54 \%$ ont été sélectionnés pour le séquençage du génome entier. Les 1131 cas séquencés ont été classés en Kappa, Alpha, Gamma et non VP/VI. Alors que les cas Alpha et Gamma se sont avérés avoir un taux d'attaque significativement plus élevé parmi les contacts familiaux par rapport aux cas non VI/VP, ce n'était pas le cas pour le variant Kappa.

Conclusion : L'analyse épidémiologique soutient la désignation du variant Kappa comme un VI et non un VP. Les variants Alpha et Gamma se sont avérés plus transmissibles, ce qui explique leur domination ultérieure dans la région et la disparition rapide du variant Kappa. Les stratégies de surveillance des variants doivent mettre l'accent à la fois sur la détection des VP établis et sur la détection de nouveaux VP potentiels.
Cette oeuvre est mise à la disposition selon les termes de la licence internationale Creative Commons Attribution 4.0

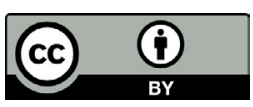

Affiliations

${ }^{1}$ Island Health, Victoria, BC

${ }^{2}$ Centre de contrôle des maladies de la Colombie-Britannique, Vancouver, BC

\section{*Correspondance :}

shaherazad.ghafari@islandhealth. ca

Citation proposée : Ghafari C, Benusic M, Prystajecky N, Sbihi H, Kamelian K., Hoang L. Analyse

épidémiologique de l'émergence et de la disparition du variant Kappa du SRAS-CoV-2 dans une région de la

Colombie-Britannique, Canada. Relevé des maladies transmissibles au Canada 2022;48(1):24-9.

https://doi.org/10.14745/ccdr.v48i01a04f

Mots-clés : SRAS-CoV-2, COVID-19, variant Kappa, variant préoccupant, variant d'intérêt, surveillance des

variants

\section{Introduction}

Le variant B.1.617 du coronavirus du syndrome respiratoire aigu sévère 2 (SRAS-CoV-2) a été désigné comme le quatrième variant préoccupant (VP) par l'Organisation mondiale de la Santé (OMS) en mai 2021 en raison des inquiétudes liées à une transmissibilité plus élevée et à une diminution potentielle de l'efficacité des traitements et des vaccins (1). Depuis, B.1.617 a été subdivisé en B.1.617.2 (Delta), qui reste un VP, et B.1.617.1 (Kappa), qui est maintenant un variant d'intérêt ( $\mathrm{VI})$ (2). L'inquiétude demeure en ce qui concerne Kappa, car un pic de cas à la fin mai 2021 a entraîné des mesures de contrôle en Australie (3). 
Dans cet article, l'épidémiologie de Kappa dans une région de la Colombie-Britannique, au Canada, du 10 mars au 2 mai 2021, est rapportée en relation avec d'autres variants circulants. L'objectif de cette analyse est de déterminer si l'épidémiologie de Kappa soutient ce déclassement en statut de VI, ou si le Kappa est similaire aux VP circulant simultanément sur le plan épidémiologique.

\section{Méthodes}

Entre le 10 mars et le 2 mai 2021, les échantillons cliniques qui ont reçu un résultat positif pour le SRAS-CoV-2 ont été soumis à un dépistage de VP par un test quantitatif d'amplification en chaîne par polymérase ciblant les mutations N501Y et E484K du spicule du gène, permettant la détection des variants Alpha, Beta et Gamma (4). S'ils donnent des résultats positifs pour N501Y, les échantillons sont présumés positifs pour Alpha; s'ils sont positifs pour N501Y et E484K, les échantillons sont présumés positifs pour Bêta et Gamma; s'ils sont négatifs pour N501Y et E484K, les échantillons ne sont pas des VP; et s'ils sont négatifs pour N501Y et positifs pour E484K, les échantillons ne sont pas des VP. Environ $30 \%$ des spécimens positifs pour le VP et $20 \%$ des spécimens négatifs pour le VP ont été sélectionnés pour le séquençage du génome entier.

Une fois que le Kappa a été détecté dans la région, le séquençage de certains échantillons supplémentaires négatifs au VP a été effectué en fonction de la géographie et des entretiens de recherche de contacts $(n=162)$. Les spécimens ont été séquencés à l'aide d'une approche de séquençage basée sur des amplicons de 1200 pb (5) sur un Illumina MiSeq ou NextSeq. Les séquences consensus SRAS-CoV-2 ont été générées à l'aide d'un pipeline Nextflow modifié pour les outils bioinformatiques de terrain du réseau ARTIC (6). Les lignées ont été attribuées à l'aide de Pangolin (version 2.4.2, pangoLEARN (7)) et les paramètres de contrôle de la qualité du séquençage ont été évalués à l'aide de nCoV-tools (version 1.5.1). Les spécimens ayant une couverture du génome supérieure à $85 \%$ et ne présentant pas de signalement de contrôle de la qualité (i.e. un excès d'ambiguïté) ont été utilisés dans les analyses suivantes. L'analyse phylogénétique a été réalisée à l'aide du projet Nextstrain, une plateforme à code source ouvert pour l'analyse et la visualisation des données génomiques. Augur version 10.2.0 et Auspice version 2.21.0 ont été utilisés respectivement pour l'analyse bio-informatique et la visualisation des données. Les séquences de consensus ont été déposées sur GISAID (Global Initiative on Sharing Avian Influenza Data).

Kappa a été comparé aux non-VP ou aux VI selon les critères de l'OMS (8) et aux principaux VP en circulation dans la région, Alpha et Gamma. Les indicateurs épidémiologiques inclus concernaient les données démographiques et les critères utilisés pour établir les VP : transmissibilité, virulence et efficacité des vaccins. L'évaluation de la transmissibilité a été limitée aux contacts familiaux afin de contrôler l'intensité et la durée variables des contacts qui ont lieu dans la collectivité. La virulence a été évaluée par l'hospitalisation dans les 14 jours suivant le prélèvement de l'échantillon et le décès attribué à la maladie à coronavirus 2019 (COVID-19) avant le 1er juin 2021. Les cas ont été classés selon leur statut vaccinal : aucune dose enregistrée, partiellement vacciné (au moins 14 jours après la $1^{\text {re }}$ dose) et complètement vacciné (au moins 14 jours après la $2^{\mathrm{e}}$ dose). Une analyse statistique a été effectuée en comparant les cas Kappa aux cas Alpha et Gamma, les cas Kappa aux cas non VPC/VI, et les cas Alpha et Gamma aux cas non VP/VI. Les tests du chi carré ou de Kruskal Wallis ont été réalisés à l'aide de STATA (version 16; StataCorp LLC), la signification statistique étant fixée à alpha $=0,05$.

\section{Résultats}

Entre le 10 mars 2021 et le 2 mai 2021, 2079 cas de COVID-19 ont été signalés dans la région Island Health; $54 \%$ des spécimens ont été sélectionnés pour le séquençage du génome entier. La proportion de spécimens envoyés pour le séquençage du génome entier est restée relativement stable tout au long de la période d'étude. La figure 1 présente une courbe épidémique de 1131 cas séquencés, classés en Kappa, Alpha, Gamma et non VP/VI. Le premier spécimen Kappa a été collecté le 10 mars 2021 et initialement détecté dans environ la moitié des cas séquencés dans la région Island Health. Alors que le nombre de cas Kappa par semaine a augmenté et atteint un pic de plus de 50 par semaine au cours des deux premières semaines d'avril 2021, la proportion relative de cas Kappa a diminué en raison de la forte augmentation relative des cas Alpha et Gamma. Au 14 juillet 2021, le dernier cas de Kappa a été signalé le 2 mai 2021. Dix-neuf cas Delta et quatre cas Bêta ont été détectés et exclus de cette analyse, car il s'agissait de VP/VI relativement rares dans la région au cours de cette période.

Le tableau 1 compare les caractéristiques des cas Kappa, Alpha et Gamma et des cas non VP/VI. La distribution des âges était similaire entre les cas Kappa et les cas non VP/VI, mais significativement différente par rapport aux cas Alpha et Gamma $(p<0,01)$. Un peu plus de la moitié des cas Kappa étaient des femmes (52,8\%), tandis que la majorité des cas Alpha et Gamma et des cas non VP/VI étaient des hommes $(53,9 \%$ et $58,2 \%$, respectivement). La source présumée était similaire pour les trois catégories de variantes, avec environ trois quarts des cas (73,0 \%-77,3\%) liés à un cas ou à un groupe de cas confirmés et le reste inconnu. Un cas de Kappa était lié à un voyage international et n'était pas responsable sur le plan épidémiologique de l'introduction de Kappa dans la région. Aucune différence significative n'a été détectée dans le taux d'attaque parmi les contacts familiaux entre Kappa et Alpha et Gamma (33,7 \% contre 37,7 \%) ou non VP/VI (33,7 \% contre $27,7 \%)$. Cependant, Alpha et Gamma ont eu un taux d'attaque statistiquement plus élevé par rapport aux non VP/VI $(37,7 \%$ contre $27,7 \%, p=0,01)$. Des proportions similaires de cas symptomatiques ont été observées dans toutes les catégories. 
Figure 1 : Nombre (A) et pourcentage (B) d'échantillons sa séquencés de cas de COVID-19 confirmés dans la région Island Health de Colombie-Britannique, Canada, du 10 mars au 2 mai 2021

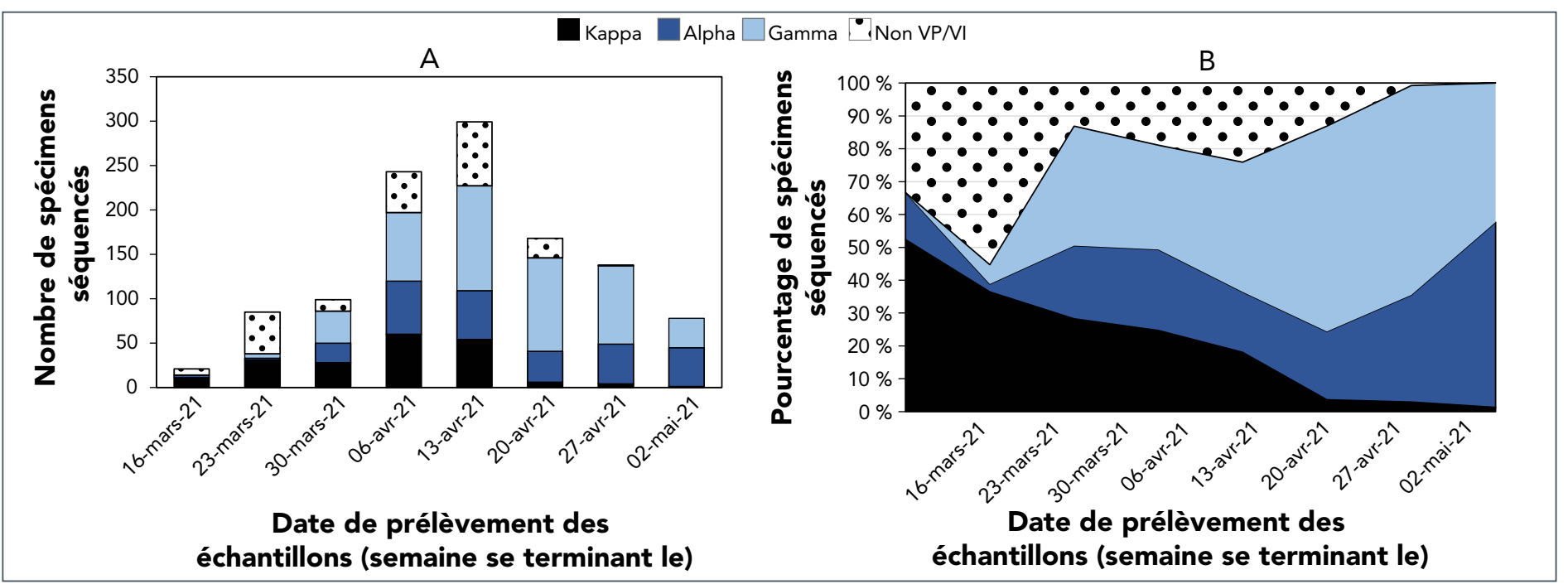

Abréviations : COVID-19, maladie à coronavirus 2019; VI, variant d'intérêt; VP, variant préoccupant

a Comme Kappa, Gamma ou non variant préoccupant/variant d'intérêt

Tableau 1 : Comparaison des caractéristiques des cas Kappa, Alpha et Gamma et des cas non variants préoccupants/variants d'intérêt dans la région Island Health de la Colombie-Britannique, Canada, du 10 mars au 2 mai 2021

\begin{tabular}{|c|c|c|c|c|c|c|c|c|c|}
\hline \multirow{3}{*}{ Caractéristiques des cas } & \multicolumn{6}{|c|}{ Variant du SRAS-CoV-2 } & \multicolumn{3}{|c|}{ valeur $\boldsymbol{p}$} \\
\hline & \multicolumn{2}{|c|}{ Kappa } & \multicolumn{2}{|c|}{ Alpha et Gamma } & \multicolumn{2}{|c|}{ Non VP/VI } & \multirow{2}{*}{$\begin{array}{l}\text { Kappa } \\
\text { contre } \\
\text { Alpha et } \\
\text { Gamma }\end{array}$} & \multirow{2}{*}{$\begin{array}{c}\text { Kappa } \\
\text { contre } \\
\text { non VP/ } \\
\text { VI }\end{array}$} & \multirow{2}{*}{$\begin{array}{c}\text { Alpha et } \\
\text { Gamma } \\
\text { contre } \\
\text { non VP/ } \\
\text { VI }\end{array}$} \\
\hline & $\mathbf{n}$ & $\%$ & $\mathbf{n}$ & $\%$ & $\mathbf{n}$ & $\%$ & & & \\
\hline \multicolumn{10}{|l|}{ Données démographiques } \\
\hline Nombre de cas (total = 1 131) & 195 & 17,2 & 728 & 64,4 & 208 & 18,4 & s.o. & s.o. & s.o. \\
\hline Âge médian en années (EI) & 34 & $21-54$ & 33 & $23-50$ & 36 & $25-56$ & 0,63 & 0,46 & 0,10 \\
\hline \multicolumn{10}{|l|}{ Groupe d'âge (années) } \\
\hline 0 à17 & 31 & 15,9 & 110 & 15,1 & 29 & 13,9 & \multirow{5}{*}{$<0,01$} & \multirow{5}{*}{0,96} & \multirow{5}{*}{$<0,01$} \\
\hline 18 à 44 & 88 & 45,1 & 382 & 52,5 & 99 & 47,6 & & & \\
\hline 45 à 64 & 46 & 23,6 & 178 & 24,5 & 51 & 24,5 & & & \\
\hline 65 à 74 & 16 & 8,2 & 46 & 6,3 & 16 & 7,7 & & & \\
\hline 75 et plus & 14 & 7,2 & 12 & 1,7 & 13 & 6,3 & & & \\
\hline \multicolumn{10}{|l|}{ Sexe } \\
\hline Femme & 103 & 52,8 & 336 & 46,2 & 87 & 41,8 & \multirow{2}{*}{0,10} & \multirow{2}{*}{0,03} & \multirow{2}{*}{0,27} \\
\hline Homme & 92 & 47,2 & 392 & 53,9 & 121 & 58,2 & & & \\
\hline \multicolumn{10}{|l|}{ Source présumée } \\
\hline Voyages internationaux & 1 & 0,6 & 1 & 0,2 & 0 & 0,0 & \multirow{3}{*}{0,34} & \multirow{3}{*}{0,58} & \multirow{3}{*}{0,39} \\
\hline Lié à un cas confirmé ou à un groupe & 131 & 73,2 & 525 & 77,3 & 138 & 73,0 & & & \\
\hline Inconnu & 47 & 26,3 & 153 & 22,5 & 51 & 27,0 & & & \\
\hline \multicolumn{10}{|l|}{ Transmissibilité } \\
\hline $\begin{array}{l}\text { Taux d'attaque parmi les contacts } \\
\text { familiaux }\end{array}$ & $67 / 199$ & 33,7 & $217 / 576$ & 37,7 & $52 / 188$ & 27,7 & 0,20 & 0,31 & 0,01 \\
\hline
\end{tabular}


Tableau 1 : Comparaison des caractéristiques des cas Kappa, Alpha et Gamma et des cas non variants préoccupants/variants d'intérêt dans la région Island Health de la Colombie-Britannique, Canada, du 10 mars au 2 mai 2021 (suite)

\begin{tabular}{|c|c|c|c|c|c|c|c|c|c|}
\hline \multirow{3}{*}{ Caractéristiques des cas } & \multicolumn{6}{|c|}{ Variant du SRAS-CoV-2 } & \multicolumn{3}{|c|}{ valeur $p$} \\
\hline & \multicolumn{2}{|c|}{ Kappa } & \multicolumn{2}{|c|}{ Alpha et Gamma } & \multicolumn{2}{|c|}{ Non VP/VI } & \multirow{2}{*}{$\begin{array}{l}\text { Kappa } \\
\text { contre } \\
\text { Alpha et } \\
\text { Gamma }\end{array}$} & \multirow{2}{*}{$\begin{array}{c}\text { Kappa } \\
\text { contre } \\
\text { non } \\
\text { VP/VI }\end{array}$} & \multirow{2}{*}{$\begin{array}{c}\text { Alpha et } \\
\text { Gamma } \\
\text { contre } \\
\text { non } \\
\text { VP/VI }\end{array}$} \\
\hline & $\mathbf{n}$ & $\%$ & $\mathbf{n}$ & $\%$ & $\mathbf{n}$ & $\%$ & & & \\
\hline \multicolumn{10}{|l|}{ Virulence } \\
\hline Symptomatique & 171 & 87,7 & 629 & 86,4 & 183 & 88,0 & 0,64 & 0,83 & 0,45 \\
\hline $\begin{array}{l}\text { Hospitalisation dans les } 14 \text { jours } \\
\text { suivant le prélèvement de l'échantillon }\end{array}$ & 3 & 1,5 & 33 & 4,5 & 8 & 3,9 & 0,06 & 0,16 & 0,67 \\
\hline Décès attribués à la COVID-19 & 1 & 0,5 & 5 & 0,6 & 1 & 0,5 & 0,79 & 0,96 & 0,64 \\
\hline \multicolumn{10}{|l|}{ Efficacité de la vaccination } \\
\hline Aucune dose enregistrée & 188 & 96,4 & 697 & 95,7 & 203 & 97,6 & \multirow{3}{*}{0,83} & \multirow{3}{*}{0,48} & \multirow{3}{*}{0,45} \\
\hline Partiellement vacciné & 7 & 3,6 & 30 & 4,1 & 5 & 2,4 & & & \\
\hline Entièrement vacciné & 0 & 0 & 1 & 0,1 & 0 & 0 & & & \\
\hline
\end{tabular}

Abréviations : COVID-19, maladie à coronavirus 2019; El, écart interquartile; SRAS-CoV-2, coronavirus du syndrome respiratoire aigu sévère 2; s.o., sans objet; VI, variant d'intérêt; VP, variant préoccupant

a 84 cas ont été exclus lorsqu'aucune catégorie d'exposition n'a été enregistrée, ce qui représente $7 \%$ à $9 \%$ de chacune des catégories de variants

Les taux d'hospitalisation n'étaient pas significativement inférieurs pour les cas Kappa par rapport aux cas Alpha et Gamma $(1,5 \%$ contre $4,5 \%, p=0,06)$. Les taux de létalité étaient faibles $(0,5 \%-0,6 \%)$ et statistiquement similaires dans tous les groupes. La majorité des cas n'étaient pas vaccinés $(95,7 \%-97,6 \%)$ et aucune différence statistique n'a été observée dans les cas d'infection post-vaccinale.
La figure 2 présente un arbre phylogénétique des cas de Kappa dans cette étude. Parmi les 195 cas, sept groupes génomiques distincts ont été établis. Les groupes génomiques reflétaient la distribution géographique des cas et correspondaient aux schémas de transmission établis par les entretiens de recherche de contacts. Les groupes génomiques ne comprenaient aucun cas provenant d'autres régions de la Colombie-Britannique. Aucun des cas des sept groupes génomiques n'avait d'antécédents de voyage documentés.

Figure 2 : Diversité génétique du SRAS-CoV-2 pour les cas Kappa dans la région Island Health de la Colombie-Britannique, Canada, du 10 mars au 2 mai 2021a

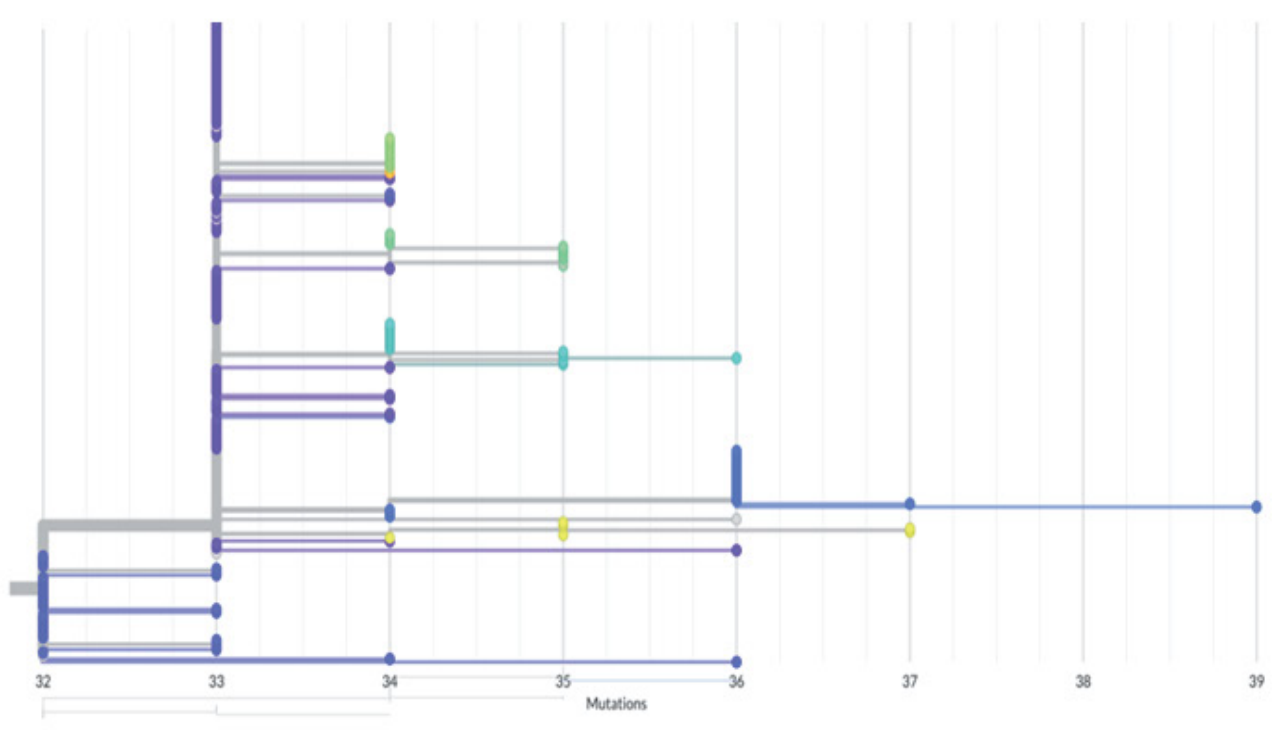

Abréviation : SRAS-CoV-2, coronavirus du syndrome respiratoire aigu sévère 2

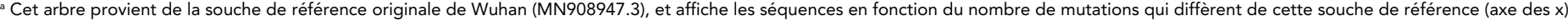
La couleur des branches est basée sur la parenté génétique, déterminée par l'algorithme Cluster Picker 


\section{Discussion}

Alors qu'il était responsable de plus d'un cas sur six pendant la période d'étude, d'environ un tiers des cas pendant les trois premières semaines de l'étude et de sept groupes génomiques et épidémiologiques distincts, Kappa a disparu de la région, le dernier cas ayant été détecté le 2 mai 2021. Au cours de la même période, Alpha et Gamma sont devenus les souches dominantes dans la région. Le fait que les variants Alpha et Gamma aient un taux d'attaque statistiquement plus élevé parmi les contacts familiaux que les variants non VP/VI suggère que, collectivement, ces variants sont plus transmissibles, ce que confirme leur désignation comme VP. Cette transmissibilité plus élevée peut expliquer pourquoi Alpha et Gamma semblent avoir surclassé Kappa, qui avait un taux d'attaque des ménages statistiquement similaire à celui des non VP/VI. Une tendance similaire a été observée au Royaume-Uni où, malgré une augmentation initiale rapide de la proportion de cas de Kappa, ce dernier est maintenant responsable de moins de $0,1 \%$ des cas récents de $\mathrm{VP} / \mathrm{VI}$ (8). En Inde, la proportion de cas séquencés était supérieure à $40 \%$ pour le variant Kappa en mars 2021, mais elle est maintenant tombée à moins de $20 \%$, le variant Delta ayant dominé (9).

Le taux d'hospitalisation plus faible des cas Kappa par rapport aux cas Alpha et Gamma observés dans cette étude correspond aux résultats du Royaume-Uni où $1,0 \%$ des cas Kappa ont été hospitalisés, contre $2,8 \%$ des cas Alpha, et où aucun des plus de 400 cas Kappa n'est décédé (10). Bien qu'aucune différence n'ait été observée entre les cas d'infection post-vaccinale du vaccin Kappa et les cas non VP/VI dans cette étude, une étude utilisant le sérum de personnes vaccinées a montré une réduction de 2,7 fois des titres de neutralisation moyens géométriques contre la variante Kappa par rapport à une souche non VP/VI (11).

Étant donné que Kappa ne circulait pas au Canada avant sa détection dans cette région de la Colombie-Britannique, on suppose qu'il a été introduit par des voyages internationaux. Les données du groupe génomique suggèrent une introduction unique de Kappa dans la région, bien qu'aucun des cas n'ait eu d'antécédents de voyage. La date de prélèvement de l'échantillon le plus ancien (10 mars 2021) est postérieure à la mise en place par le gouvernement canadien de tests et de quarantaine obligatoires pour les voyageurs de retour au pays (22 février 2021). II est possible que l'introduction initiale ait précédé l'introduction de ce programme et qu'elle n'ait pas été captée par les tests de routine.

\section{Limites}

Cette étude ne comporte probablement pas assez de candidats, ce qui pose un défi pour déterminer les petites différences entre les cas Kappa, Alpha et Gamma, et les cas non VP/VI. Au cours de la période d'étude, il y a également eu une couverture élevée de la première dose de vaccin ARNm chez les plus vulnérables (en particulier les personnes âgées de plus de 70 ans), et une couverture globale de la première dose de vaccin qui est passée de $\sim 10 \%$ à $25 \%$ pour les adultes de la région, ce qui a probablement réduit le risque relatif d'infection et de gravité chez les personnes vulnérables, réduisant ainsi la puissance de cette étude pour fournir des distinctions entre les catégories de variants.

\section{Conclusion}

Il est difficile de tirer des conclusions concernant la virulence et l'efficacité du vaccin, car les hospitalisations, les décès et les cas d'infection post-vaccinale sont des événements relativement rares. Cependant, l'épidémiologie de Kappa dans la région Island Health de Colombie-Britannique, au Canada, appuie l'affirmation de I'OMS selon laquelle Kappa ne répond pas aux critères du VP.

Enfin, la surveillance des variants décrite ici souligne l'importance de réaliser un séquençage du génome entier sur au moins une partie des cas qui sont catégorisés comme non VP sur la base de la réaction en chaîne par polymérase des mutations N501Y et E484K. Sans le séquençage du génome entier de ces échantillons, il est peu probable que Kappa ou Delta auraient été décelés dans la région, ce qui a guidé le séquençage du génome entier ciblé sur la base des liens épidémiologiques pour surveiller la propagation. Une stratégie similaire de surveillance des variants pourrait être utilisée dans le but de maintenir la détection des variants qui ne partagent pas les mutations communes trouvées dans les variants Alpha, Bêta et Gamma.

\section{Déclaration des auteurs}

C. G. et M. B. - Analyse des données épidémiologiques N. P., H. S., K. K., L. H. - Analyse des données génomiques

Tous les auteurs ont contribué à la conceptualisation, à la méthodologie et à la rédaction du manuscrit.

Le contenu de l'article et les points de vue qui y sont exprimés dans cet article n'engagent que les auteurs et ne correspondent pas nécessairement à ceux du gouvernement du Canada.

\section{Intérêts concurrents}

Aucun.

\section{Remerciements}

L'équipe de surveillance de la gestion des cas et des contacts de Island Health et la Dre S. Allison ont participé à la gestion des cas mentionnés dans cette étude. Le personnel du laboratoire de santé publique du Centre de contrôle des maladies de la Colombie-Britannique a effectué le qPCR et le séquençage du variant préoccupant (VP) des échantillons 
cliniques du coronavirus du syndrome respiratoire aigu sévère 2 (SRAS-CoV-2). La Dre A. Jassem et le Dr J. Tyson ont contribué au dépistage des VP et aux tests de séquençage du génome entier.

\section{Financement}

Aucun.

\section{Références}

1. World Health Organization. Weekly epidemiological update on COVID-19 - 11 May 2021. Geneva (CH): WHO; May 2021 (accédé 2021-07-21). https://www.who.int/publications/m/ item/weekly-epidemiological-update-on-covid-19---11may-2021

2. World Health Organization. Weekly epidemiological update on COVID-19 - 1 June 2021. Geneva (CH): WHO; June 2021 (accédé 2021-07-21). https://www.who.int/publications/m/ item/weekly-epidemiological-update-on-covid-19---1june-2021

3. Australian Government Department of Health. Greater Melbourne declared a hotspot for Commonwealth support. Canberra, Australia, AGDH; May 2021 (accédé 2021-07-21). https://www.health.gov.au/news/greater-melbournedeclared-a-hotspot-for-commonwealth-support

4. Hogan CA, Jassem AN, Sbihi H, Joffres $Y$, Tyson JR, Noftall K, Taylor M, Lee T, Fjell C, Wilmer A, Galbraith J, Romney MG, Henry B, Krajden M, Galanis E, Prystajecky N, Hoang LM. Rapid Increase in SRAS-CoV-2 P.1 Lineage Leading to Codominance with B.1.1.7 Lineage, British Columbia, Canada, January-April 2021. Emerg Infect Dis 2021;27(11):2802-9. DOl PubMed

5. Freed NE, Vlková M, Faisal MB, Silander OK. Rapid and inexpensive whole-genome sequencing of SRAS-CoV-2 using $1200 \mathrm{bp}$ tiled amplicons and Oxford Nanopore Rapid Barcoding. Biol Methods Protoc. 2020;5(1):bpaa014. DOI
6. Galloway SE, Paul P, MacCannell DR, Johansson MA, Brooks JT, MacNeil A, Slayton RB, Tong S, Silk BJ, Armstrong GL, Biggerstaff M, Dugan VG. Emergence of SRAS-CoV-2 B.1.1.7 Lineage - United States, December 29, 2020-January 12, 2021. MMWR Morb Mortal Wkly Rep 2021;70(3):95-9. DOI PubMed

7. Rambaut A, Holmes EC, O’Toole Á, Hill V, McCrone JT, Ruis C, du Plessis L, Pybus OG. A dynamic nomenclature proposal for SRAS-CoV-2 lineages to assist genomic epidemiology. Nat Microbiol 2020;5(11):1403-7. DOI PubMed

8. Organisation mondiale de la Santé. Suivi des variants du SRAS-CoV-2. Genève (CH) : OMS (modifié 2021-12; accédé 2021-07-21). https://www.who.int/fr/activities/trackingSRAS-CoV-2-variants/tracking-SRAS-CoV-2-variants

9. Singh J, Rahman SA, Ehtesham NZ, Hira S, Hasnain SE. SRAS-CoV-2 variants of concern are emerging in India. Nat Med 2021;27(7):1131-3. DOI PubMed

10. Public Health England. SRAS-CoV-2 variants of concern and variants under investigation in England. Technical briefing 15. London (UK): PHE; June 2021 (accédé 2021-07-21). https://assets.publishing.service.gov.uk/government/ uploads/system/uploads/attachment_data/file/993879/ Variants_of_Concern_VOC_Technical_Briefing_15.pdf

11. Liu C, Ginn HM, Dejnirattisai W, Supasa P, Wang B, Tuekprakhon A, Nutalai R, Zhou D, Mentzer AJ, Zhao Y, Duyvesteyn HM, López-Camacho C, Slon-Campos J, Walter TS, Skelly D, Johnson SA, Ritter TG, Mason C, Costa Clemens SA, Gomes Naveca F, Nascimento V, Nascimento F, Fernandes da Costa C, Resende PC, Pauvolid-Correa A, Siqueira MM, Dold C, Temperton N, Dong T, Pollard AJ, Knight JC, Crook D, Lambe T, Clutterbuck E, Bibi S, Flaxman A, Bittaye M, Belij-Rammerstorfer S, Gilbert SC, Malik T, Carroll MW, Klenerman P, Barnes E, Dunachie SJ, Baillie V, Serafin N, Ditse Z, Da Silva K, Paterson NG, Williams MA, Hall DR, Madhi S, Nunes MC, Goulder P, Fry EE, Mongkolsapaya J, Ren J, Stuart DI, Screaton GR. Reduced neutralization of SRAS-CoV-2 B.1.617 by vaccine and convalescent serum. Cell 2021;184(16):4220-4236.e13. DOI PubMed 